\title{
The Phenotypic Conversion of Macrophage Detected by FACS in EAE Mice Treated with or without Fasudil ${ }^{*}$
}

\author{
Chunyun Liu ${ }^{1}$, Yong Xie ${ }^{1}$, Yanhua $\mathrm{Li}^{1}$, Jiezhong $\mathrm{Yu}^{1}$, Ling Feng ${ }^{1}$, Shaowei Hou ${ }^{1}$, \\ Haifei Zhang ${ }^{1}$, Cungen Ma ${ }^{1 /}$, Baoguo Xiao" ${ }^{2 \#}$ \\ ${ }^{1}$ Institute of Brain Science, Department of Neurology, Medical School, Shanxi Datong University, Datong, China \\ ${ }^{2}$ Institute of Neurology, Huashan Hospital, Fudan University, Shanghai, China \\ Email: \#macungen2001@yahoo.com.cn
}

Received 2013

\begin{abstract}
We studied the changes of macrophage populations in splenic mononuclear cells of experimental autoimmune encephalomyelitis (EAE) mice treated with or without Fasudil. Phenotypic analysis using flow cytometry showed that the levels of TLR4, CD11c and CD40 which represent the type 1 macrophage, were depressed in Fasudil-treated mice. In contrast, it was observed the expressions of CD200 and CD14 which typify the type 2 macrophage were elevated in Fasudil-treated mice as compared to EAE mice. And we also found that Fasudil at dose of $40 \mathrm{mg} / \mathrm{kg}$ alleviated the severity of symptom in EAE mice. Based on the evidence that M1 macrophages are neurotoxic and M2 macrophages promote a regenerative growth, indicating that polarization and shifting of macrophages into M2 cells may also play key roles in treatment of EAE.
\end{abstract}

Keywords: Experiment Autoimmune Excephomeylitis; Fasudil; Flow Cytometry; Macrophage

\section{Introduction}

Multiple sclerosis (MS) is a chronic inflammatory demyelinating disease of the central nervous system (CNS) that afflicts approximately 1.4 million people worldwide [1]. Experimental autoimmune encephalomyelitis (EAE) is an animal model of MS, which exhibits some of the symptomatology and pathology observed in MS patients. The immunopathogenesis of EAE involves the disruption of the BBB and the integrated attack of T cells, macrophages, and dendritic cells [2].

Fasudil (1-(5-isoquinolinesulfonyl)-homo-piperazine), a selective Rho-kinase (ROCK) inhibitor, has been used to treat cerebral vasospasm and stroke. In EAE model, the clinical severity of EAE is tightly related to the infiltration of inflammatory macrophages and $\mathrm{T}$ cells in the CNS $[3,4]$. In the present study, we observed therapeutic potential of fasudil and explored action mechanisms in EAE.

\section{Materials and Methods}

\subsection{Animals}

Female C57BL/6 mice, 8 - 10 weeks old, weight 20 - 22

*This work was supported by National Natural Science Foundation of China (No. 81070957).

\#Corresponding author. g, were purchased from Vital River Laboratory Animal Technology Co. Ltd. (Beijing, China). All experiments were conducted in accordance with the guidelines of the International Council for Laboratory Animal Science. The study was approved by the Ethics Committee of Shanxi Datong University, Datong, China. All mice were housed under pathogen-free conditions, received food and water ad libitum, and maintained in a reversed 12:12 hours (h) light/dark cycle in a temperature-controlled room $\left(25^{\circ} \mathrm{C} \pm 2^{\circ} \mathrm{C}\right)$ for one week prior to experimental manipulation.

\subsection{Induction and Clinical Evaluation of EAE in C57BL/6 Mice}

Mouse myelin oligodendrocyte glycoprotein peptide $35-55$ $\left(\mathrm{MOG}_{35-55}\right)$ was produced in an automatic synthesizer (CL. Bio-Scientific. Company, Xian, China), aminophenol sequence for $\mathrm{MOG}_{35-55}$ is MEVGWYRSPFSRVVHLYRNGK. The purity of the peptide was $>95 \%$ as determined by HPLC.The Chronic EAE was induced by subcutaneous immunization on the upper dorsal flanks with $300 \mu \mathrm{g}$ of $\mathrm{MOG}_{35-55}$ in complete Freund's adjuvant (Sigma, USA) supplemented with $3 \mathrm{mg} / \mathrm{ml}$ of $M$. Tuberculosis H37Ra (BD Difco, USA) (400 $\mu \mathrm{g} /$ mice). Mice were injected with $400 \mathrm{ng}$ of pertussis toxin (Enzo Life Sciences, USA) via abdominal cavity at the same time of 
immunization and again 48 hours later. Animals were weighed and evaluated for clinical score every other day in a blinded fashion by at least two investigators. Clinical scores of EAE were graded according to the following criteria: 0 . healthy; 1 . limp tail; 2 . ataxia and/or paresis of hindlimbs; 3. paralysis of hindlimbs and/or paresis of forelimbs; 4. tetraparalysis; 5 . moribund or death. All experiments were repeated 3 - 4 times.

\subsection{Treatment of Fasudil}

Fasudil (TIANJIN CHASE SUN PHARMACEUTICAL CO., LTD) dissolved in normal saline (NS) was injected intraperitoneally (i.p.) at $40 \mathrm{mg} / \mathrm{kg} / \mathrm{d}$ on day 3 post-immunization (p.i.) (fasudil early treatment) or at onset of EAE (fasudil late treatment) till day 28 p.i. The injection of NS was set up as control (EAE group) in similar manner.

\subsection{Preparation of Mononuclear Cells}

At day 28 p.i., mice were sacrificed and spleens were removed under aseptic conditions. MNCs from spleens were prepared by grinding the organ through a $40 \mathrm{~mm}$ nylon mesh in medium. Erythrocytes in the suspensions were osmotically lysed. Cells were then washed 3 times and re-suspended in medium. Cells were adjusted to $3 \times$ $10^{6} \mathrm{ml}$.

\subsection{Flow Cytometry (FACS)}

For phenotypic analysis, MNCs were stained for $20 \mathrm{~min}$ at room temperature in 1\% BSA-PBS buffer with the following antibodies: PE-TLR4, PE-CD40, Percp-cy5CD11c, Percp-cy5-CD200, Percp-cy5-CD14, PE-CD23. (BD Biosciences, San Diego, CA). Cells were gated using forward and sideward scatter characteristics and at least 10,000 gated events were collected using FACS Calibur flow cytometer (BD Biosciences, USA). Data were analyzed using CellQuest software.

\subsection{Statistical Analysis}

Data were presented as mean \pm S.E.M. Body weight, clinical scores and incidence were performed using the two-tailed Student's t test and were analyzed by one-way ANOVA using GraphPad Prism 4 (GraphPad Software, Inc.). The multiple comparisons were performed by the two-way ANOVA using GraphPad Prism 4. A statistically significant difference was assumed at $\mathrm{P}<0.05$.

\section{Results}

\subsection{Fasudil Alleviated the Severity of Symptom in EAE Mice}

As shown in Table 1, the incidence of EAE model was
Table 1. The clinical symptoms of mice in each group.

\begin{tabular}{ccccc}
\hline Group & n & $\begin{array}{c}\text { Incidence } \\
\text { (\%) }\end{array}$ & $\begin{array}{c}\text { Mean onset } \\
\text { date }\end{array}$ & $\begin{array}{c}\text { Mean score of } \\
\text { maximal symptom }\end{array}$ \\
\hline EAE control & 14 & 100 & $12.57 \pm 1.55$ & $2.50 \pm 1.13$ \\
Fasudil late & 14 & 78.6 & $13.45 \pm 2.07$ & $1.36 \pm 1.06^{\mathrm{b}}$ \\
Fasudil early & 14 & $28.6^{\mathrm{a}}$ & $16.50 \pm 4.20^{\mathrm{a}}$ & $0.32 \pm 0.70^{\mathrm{a}}$ \\
\hline
\end{tabular}

${ }^{\mathrm{a}} \mathrm{p}<0.05$ vs. EAE control; ${ }^{\mathrm{b}} \mathrm{p}<0.05$ vs. EAE control.

$100 \%$, while the incidence of Fasudil early treatment and late treatment was $78.6 \%$ and $28.6 \%$ ( $\mathrm{P}<0.05$ ), respectively. Mean onset was $12.37 \pm 1.55$ p.i. in EAE control, $13.45 \pm 2.07$ p.i. in fasudil early treatment and $16.50 \pm$ 4.20 p.i. in Fasudil late treatment $(\mathrm{P}<0.05)$. In early treatment, Fasudil delayed the onset of sign and dramatically reduced clinical symptoms. In late treatment, Fasudil also ameliorated clinical scores in EAE mice, as compared with EAE controls (Figure 1(A), P $<0.05$ ), In addition, the improvement of EAE clinical scores were accompanied by a decrease in body weight from days 10 to 28 p.i. (Figure 1(B)), providing further evidence that Fasudil exhibits a therapeutic potential for future clinical application.

\subsection{Fasudil Shifts M1 to M2}

To characterize the changes of macrophage populations in spleen on EAE, we assessed the expression of TLR4, CD40, CD11c, CD200, CD23 and CD14 using FACS. Phenotypic analysis of macrophage showed that the levels of TLR4, CD11c and CD40 which represent the type 1 macrophage (M1), were significantly depressed in Fasudil late treatment mice compared with EAE control (Figure 2(A), p $<0.001$ for TLR4, p $<0.05$ for CD11c and $\mathrm{p}<0.001$ for CD40). The expression of CD11c and CD40 were significantly reduced in Fasudil early treatment mice compared with EAE control (Figure 2(A), p < 0.001 for CD11c and $\mathrm{p}<0.05$ for CD40). As expected, we found that the augmented tendency of CD200, CD23 and CD14 which labeled the type 2 macrophage (M2) was observed in Fasudil treatment mice, especially in Fasudil early treatment mice (Figure 2(B)). Mice in fasudil early treatment exhibited increased expression of CD200 and CD14 in comparison to EAE control mice (P $<0.01$ and $\mathrm{P}<0.001$, respectively), whereas the elevation of CD200 expression in Fasudil late treatment mice was not obvious $(\mathrm{P}>0.05)$. Our results indicate that Fasudil treatment can shift macrophages from M1 to M2 phenotype.

\section{Discussion}

FACS was developed in 1971 by Leonard Herzenberg's team at Stanford University, which was widely used in the world for clinical and experiment research. Under 

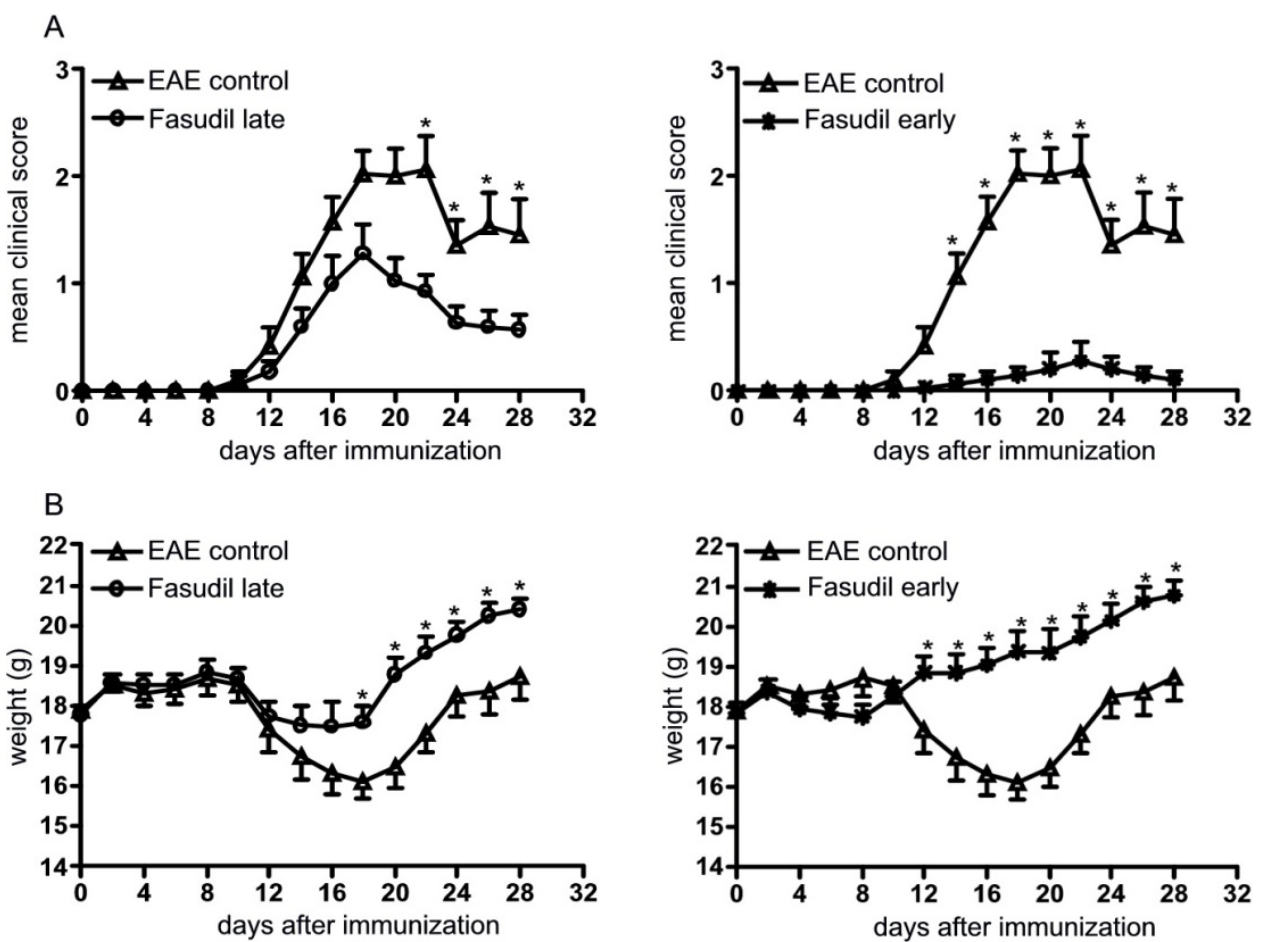

Figure 1. Fasudil treatment suppresses MOG-induced EAE. (A) Fasudil could prevent the severity of clinical symptoms to EAE, and there were significant differences of clinical symptoms score between EAE control and fasudil late treatment group on days 24 - 28 p.i. ( $* P$ < 0.017) and between EAE control and fasudil early treatment group on days $14-28$ p.i. (*P < 0.017). (B) EAE control group had more marked loss of body weights as compared with that of fasudil late treatment group on days 18 - 28 p.i. (*P $<0.05)$ and that of fasudil early treatment group on days $12-28$ p.i. $(* \mathbf{P}<0.05)$. Data are presented as mean \pm S.E.M.

continuous development, this technology has been enable to perform single cell multiparametric analysis and stem cells sorting, which will open a new way to FACS uses in the future $[5,6]$. By using FACS assay, we further explore the therapeutic mechanisms of Fasudil in treatment of EAE.

In the present study, Fasudil treatment delayed onset of EAE, and ameliorated clinical scores. The known beneficial effects of Fasudil on EAE could partly be explained by inhibition of the GTPase Rho, which resulted in a Th2 shift acting on $\mathrm{T}$ cells [7]. In addition, T-cell proliferation specific to MOG (35-55) was markedly reduced, together with a significant down-regulation of interleukin (IL)-17, IL-6, and MCP-1. In contrast, secretion of IL-4 was increased, and IL-10 was slightly elevated [4]. Our results demonstrate that Fasudil inhibited TLR4, CD11c, CD40 expression and increased CD200, CD23, CD14 expression on MNCs.

Macrophages play an important role in innate and adaptive immunity and are a heterogeneous cell population of the myeloid linage derived from monocytes. These cells show two different polarization states, M1 and M2 macrophages in response to different immune responses. It is postulated that the development of autoimmune diseases may be mediated by activating ma- crophages to a cytolytic M1 phenotype and by suppressing the activation of proregenerative macrophages to an M2 phenotype. Different phenotype and function of macrophages can be distinguished using M1 markers such as TLR4, CD11c and CD40 as well as M2 markers such as CD14, CD23 and CD200. To our knowledge, CD40 positive microglia were observed in the CNS of marmoset monkeys with acute EAE, a newly described nonhuman primate model for MS, which is important for the infiltration and retention of inflammatory leukocytes into the CNS [8]. TLR4 and CD11c could provoke antigen presenting cells to activate autoreactive immune cells and induce Ag-loaded dendritic cells to produce a marked increase in inflammatory cytokine secretion $[9,10]$. These molecules play the critical roles in driving the immune response necessary to develop EAE, and therefore, the down-regulation of TLR4, CD11c and CD40 which reveals the inhibition of proinflammatory M1 macrophages could have a direct consequence on EAE progression. CD200 is a non-signaling glycoprotein that belongs to the Ig superfamily and has been shown to inhibit immune stimulatory responses [11]. Our findings indicate that Fasudil ameliorates the development of EAE at least partially through sifting inflammatory M1 to anti-inflammatory M2 macrophages. 
"M1" phenotype
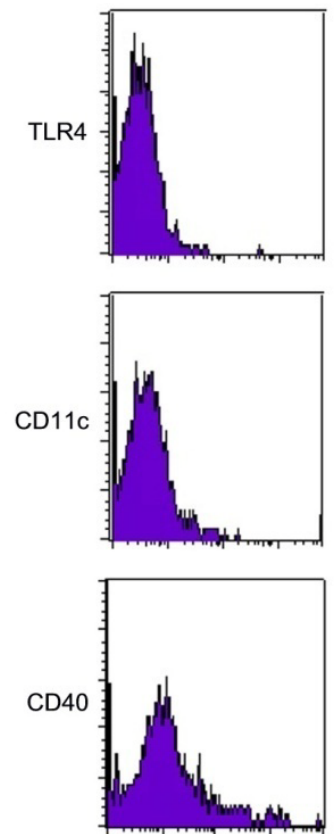

Mear EAE control

Mean Fluorescence Intensities (MF
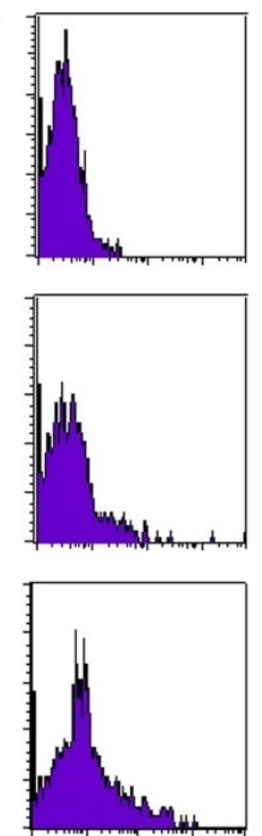

Fasudil late

"M2" phenotype
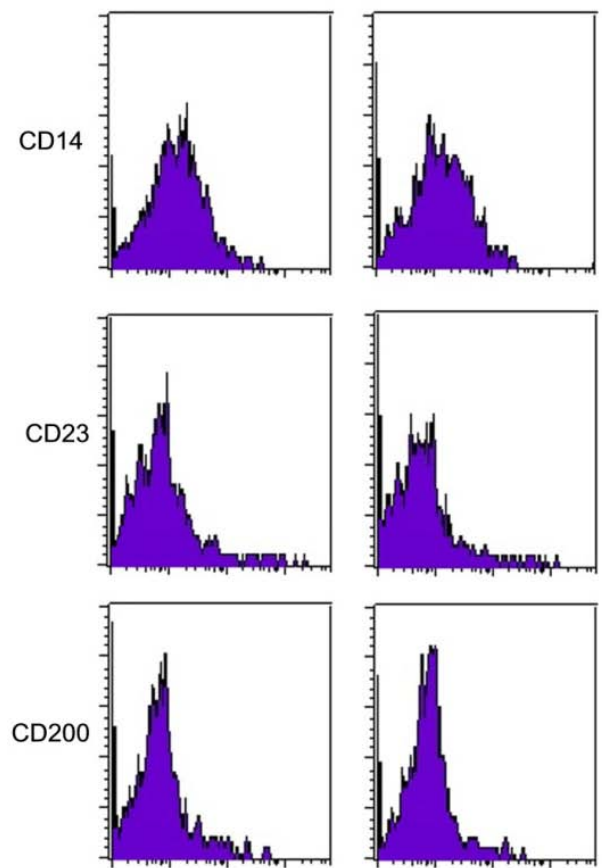

Mean Fluorescence Intensities (MF

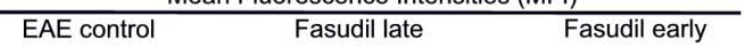

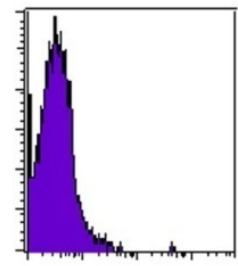
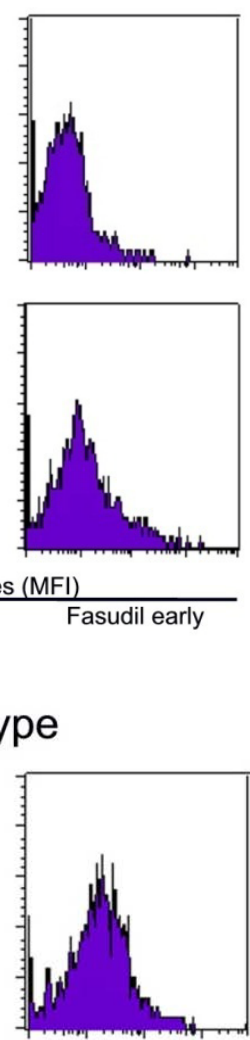

A
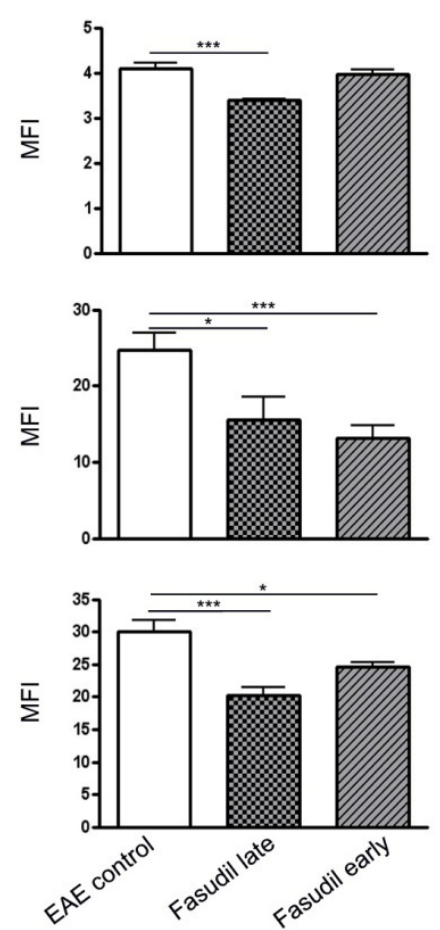

B
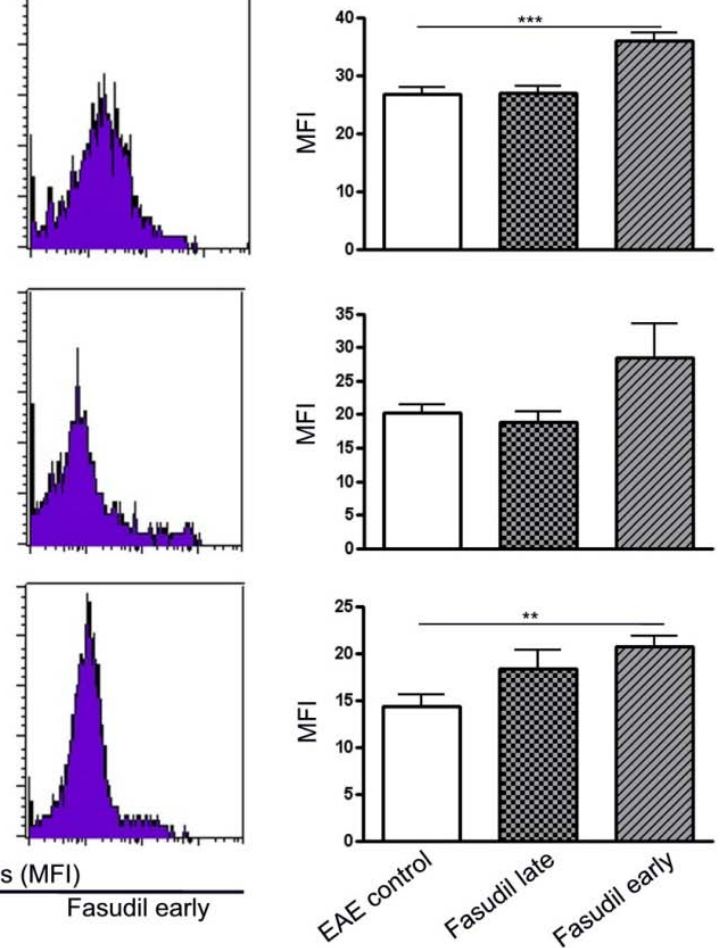

Figure 2. Effects of fasudil on splenic macrophages. The level of TLR4, CD40, CD11c, CD200, CD23 and CD14 were shown by blue histograms. It is noteworthy that Fasudil could downregulate the expression of TLR4, CD11c, CD40 and upregulate the expression of CD200, CD14. Representative histograms of three groups are shown. p-Values are indicated by asterisks as follows: $* \mathbf{P}<0.05, * * \mathbf{P}<0.01$ and $* * * \mathbf{P}<0.001$. 
In conclusion, the therapeutic effect of Fasudil in EAE may be related to shifting of macrophages from M1 to M2 phenotype. By using FACS assay, the treatment of Fasudil inhibits M1 macrophages, and elevates M2 macrophages, providing novel mechanism of Fasudil in the treatment of EAE.

\section{REFERENCES}

[1] E. M. Frohman, M. K. Racke and C. S. Raine, "Multiple Sclerosis-The Plaque and Its Pathogenesis," The New England Journal of Medicine, Vol. 254, 2006, pp. 942955. http://dx.doi.org/10.1056/NEJMra052130

[2] R. J. Winquist, A. Kwong, R. Ramachandran and J. Jain, “The Complex Etiology of Multiple Sclerosis,” Biochemical Pharmacology, Vol. 74, 2007, pp. 1321-1329. http://dx.doi.org/10.1016/j.bcp.2007.04.026

[3] O. Neuhaus, O. Stüve, S. S. Zamvil and H. P. Hartung, "Are Statins a Treatment Option for Multiple Sclerosis?" The Lancet Neurology, Vol. 3, 2004, pp. 369-371. http://dx.doi.org/10.1016/S1474-4422(04)00770-7

[4] X. Sun, M. Minohara, H. Kikuchi, T. Ishizu, M. Tanaka and H. Piao, et al., "The Selective Rho-Kinase Inhibitor Fasudil Is Protective and Therapeutic in Experimental Autoimmune Encephalomyelitis,” Journal of Neuroimmunology, Vol. 180, 2006, pp. 126-134. http://dx.doi.org/10.1016/j.jneuroim.2006.06.027

[5] Y. Gernez, L. A. Herzenberg, L. A. Herzenberg and R. Tirouvanziam, "Phospho-FACS: A Powerful Tool for Exploring Intracellular Transduction Cascades,” Revue des Maladies Respiratoires, Vol. 24, 2007, pp. 955-964. http://dx.doi.org/10.1016/S0761-8425(07)92761-8

[6] T. W. Tung, K. Heydary, R. Tirouvanziam, B. Sahaf, D. R. Parks and L. A. Herzenberg, "Modern Flow Cytometry:
A Practical Approach,” Clinics in Laboratory Medicine, Vol. 27, 2007, pp. 453-468.

http://dx.doi.org/10.1016/j.cll.2007.05.001

[7] A. A. Pineda, M. Minohara, N. Kawamura, T. Matsushita, R. Yamasaki, X. Sun, et al., "Preventive and Therapeutic Effects of the Selective Rho-Kinase Inhibitor Fasudil on Experimental Autoimmune Neuritis," Journal of the Neurological Sciences, Vol. 306, 2011, pp. 115-120. http://dx.doi.org/10.1016/j.jns.2011.03.031

[8] E. N. Benveniste, V. T. Nquyen and D. R. Wesemann, "Molecular Regulation of CD40 Gene Expression in Macrophages and Microglia," Brain, Behavior, and Immunity, Vol. 18, 2004, pp. 7-12. http://dx.doi.org/10.1016/j.bbi.2003.09.001

[9] N. A. Wolf, T. K. Amouzeqar and R. H. Swanborq, "Synergistic Interaction between Toll-Like Receptor Agonists Is Required for Induction of Experimental Autoimmune Encephalomyelitis in Lewis Rats," Journal of Neuroimmunology, Vol. 185, 2007, pp. 115-122. http://dx.doi.org/10.1016/j.jneuroim.2007.02.001

[10] S. Sinha, L. Miller, S. Subramanian, O. J. McCarty, T. Proctor, R. Meza-Romero, et al., "Binding of Recombinant T Cell Receptor Ligands (RTL) to Antigen Presenting Cells Prevents Upregulation of CD11b and Inhibits T Cell Activation and Transfer of Experimental Autoimmune Encephalomyelitis,” Journal of Neuroimmunology, Vol. 225, 2010, pp. 52-61. http://dx.doi.org/10.1016/j.jneuroim.2010.04.013

[11] S. G. Meuth, O. J. Simon, A. Grimm, N. Melzer, A. M. Herrmann, P. Spitzer, et al., "CNS Inflammation and Neuronal Degeneration Is Aggravated by Impaired CD200CD200R-Mediated Macrophage Silencing," Journal of Neuroimmunology, Vol. 194, 2008, pp. 62-69. http://dx.doi.org/10.1016/j.jneuroim.2007.11.013 